\title{
Sparse Model of the Lift Gains \\ of a Circulation Control Wing \\ with Unsteady Coanda Blowing
}

\author{
Richard Semaan, M. Yosef EI Sayed and Rolf Radespiel
}

\begin{abstract}
The present study investigates and models the lift gains and losses generated by the superposition of a periodic actuation component onto a steady component on an airfoil with a highly deflected Coanda flap. The periodic actuation is provided by two synchronized specially-designed valves that deliver actuation frequencies up to $30 \mathrm{~Hz}$ and actuation amplitudes up to $20 \%$ of the mean blowing intensity. The lift gains/losses response surface is modeled using a data-driven sparse identification approach. The results clearly demonstrate the benefits of superimposing a periodic component onto the steady actuation component for a separated or partially-attached flow, where up to $\Delta C_{l}=0.47$ lift increase is achieved. On the other hand, this same superimposition for an attached flow is detrimental to the lift, with up to $\Delta C_{l}=-0.3$ lift reduction compared to steady actuation with similar blowing intensity is observed.
\end{abstract}

Keywords High-lift $\cdot$ Sparse modeling $\cdot$ Coanda actuation

\section{Introduction}

Active flow control (AFC) with periodic actuation has been proven to bring aerodynamic benefits (e.g. Greenblatt and Wygnanski [1]; Barros et al. [2]; and Chabert et al. [3]). These benefits stem from the power reductions obtained compared to steady actuation. The success of periodic actuation is rooted in its exploitation of the flow

R. Semaan ( $ه) \cdot$ M. Y. El Sayed · R. Radespiel

Technische Universität Carolo-Wilhelmina zu Braunschweig,

Hermann-Blenk-Str. 37, 38108 Braunschweig, Germany

e-mail: r.semaan@tu-bs.de

URL:https://www.tu-braunschweig.de/ism 
instabilities, contrary to steady actuation which attempts to alter the flow topology by momentum injection.

For aircraft, circulation control in combination with high-lift devices offers several advantages over traditional high-lift configurations. The basic concept of circulation control involves the Coanda principle, where energy is introduced into the flow by means of a thin jet ejected tangentially from a slit near the trailing edge. The main advantage of circulation control is an increased lift output, which makes shorter takeoffs and landings possible. This technology was first patented by Davidson [4] in 1960 and has since been repeatedly investigated (Lachmann [5]; Wood and Nielson [6]; and Englar [7]). A circulation control wing (CCW) with steady jets, even at very small mass flow rates, has been shown to yield lift coefficients that are comparable or superior to conventional high-lift systems (Sexstone et al. [8]; Smith [9]). A particular variant of circulation control is the Coanda flap, where the objective is to keep the flow attached over a highly deflected flap by blowing a jet tangentially over its specially designed upper surface. This concept has been previously investigated and geometrically optimized in several previous studies [10-12].

Efficiency requirements demand that the lift gained through the use of circulation control be as large as possible in comparison to the momentum coefficient of the blown jet, which is usually acquired by engine bleed. This ratio is referred to as the lift gain factor. An increase in the lift gain factor can be achieved through periodic blowing. Two studies during the mid-1970s investigated pulsed blowing associated with circulation control (Oyler and Palmer [13]; Walters et al. [14]). Results from these experiments indicated that pulsed blowing reduced the mass requirements for CCW. More recently, periodic blowing on a circulation control wing with circular trailing edge was examined (Jones et al. [15]), where a 50\% reduction in the required mass flow for a required lift coefficient was achieved. It is worth to note that the benefits of periodic excitation targeting flow instabilities have also been demonstrated in other flow control applications, such as pulsed actuation over a flap (e.g. Petz and Nitsche [18]) and acoustic excitation (e.g. Greenblatt and Wygnanski [1]; Seifert et al. [19]).

In this study, we investigate and model the lift gains/losses from superimposed steady and periodic Coanda actuation. The investigation is based on experimental measurements in the large water tunnel facility at the Technische Universität Braunschweig using the configuration studied by Burnazzi and Radespiel [20] as part of the Coordinated Research Centre 880 (CRC 880). The periodic actuation is achieved using two specially-designed high-speed proportional valves. The results demonstrate the benefits of superimposing a periodic actuation component onto the steady one, where up to $\Delta C_{l}=0.47$ lift increase compared to the corresponding steady blowing is observed. 


\section{Experimental Setup}

\subsection{Model}

The experimental 2D model is a modified DLR-F15 airfoil with a $c=300 \mathrm{~mm}$ chord length and a $1 \mathrm{~m}$ span. It features a highly deflected Coanda-flap and a drooped leading edge, as seen in Fig. 1 . The Coanda-flap has a length of $c_{f l}=0.25 \cdot c$ (i.e. $c_{f l}=$ $75 \mathrm{~mm}$ ) and is deflected by $65^{\circ}$ for a landing configuration. The Coanda jet is blown over the flap shoulder through a $0.00067 \cdot c$ slit following design considerations presented in Radespiel et al. [12]. The jet is supplied through a plenum inside the model, which is connected on both sides of the model to a KSB Movitec VF 32-7 PD multi-stage inline-pump installed outside the tunnel that delivers flow rates up to $101 / \mathrm{s}$ at 8 bar pressure.

The droop nose shape was reached through a parametric study that maximized the lift [20]. The geometry is morphed from the clean nose by deflecting the leading edge down by $90^{\circ}$ over a length of $0.2 c$ and increasing the leading edge thickness by $60 \%$. The reference coordinate system, shown in Fig. 1, is that of the clean airfoil, where the leading edge coincides with the origin. Henceforth, all subsequent dimensions are with respect to this reference coordinate system.

\subsection{Facilities}

The experiment is carried out in the large water tunnel facility (Großer Wasserkanal Braunschweig (GWB)) at the Technische Universität Braunschweig [21] (Fig. 1b). The facility is a Göttingen-type closed return tunnel, with a $6 \mathrm{~m}$ long and $1 \mathrm{~m} \times 1 \mathrm{~m}$ test section. The flow is driven by a $1.5 \mathrm{~m}$ diameter one-stage axial pump powered by a variable frequency drive $160 \mathrm{~kW}$ electric motor, yielding flow velocities up to $6 \mathrm{~m} / \mathrm{s}$ in the test section. To inhibit cavitation, the tunnel can be pressurized up to 2 bar above ambient pressure.

The choice of a water tunnel is based on time scale considerations. Due to the much smaller kinematic viscosity of water compared to air (about 1/16th that of air

(a)
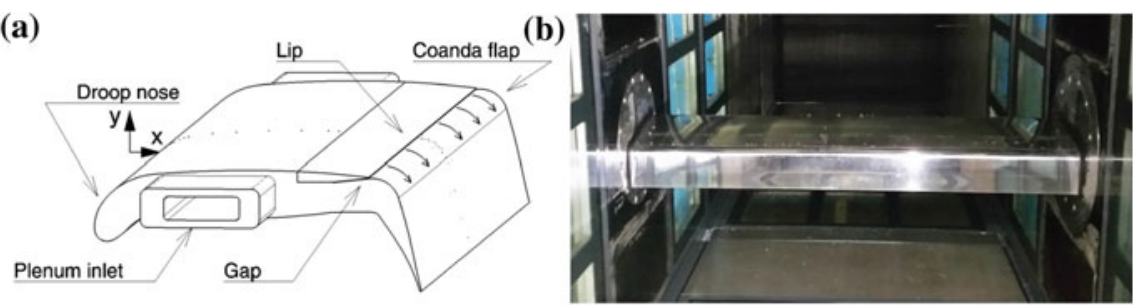

Fig. 1 a Schematic and $\mathbf{b}$ a picture of the experimental model installed in the water tunnel 
at room temperature), the same Reynolds number for a given model size is reached at a fraction of the free stream velocity. Higher Reynolds numbers can be achieved by heating the water (up to $40^{\circ} \mathrm{C}$ are possible in the GWB). Therefore, it is possible in a water tunnel to capture a considerably larger portion of the flow dynamics than in air at similar Reynolds number, since flow phenomena happen on larger time scales. The larger time scales also make water tunnels better suited for closed-loop flow control experiments, which would be the focus of our future efforts.

Although Reynolds numbers of up to $\operatorname{Re}=\frac{V_{\infty} c}{\nu_{\infty}}=2.5 \times 10^{6}$ are possible, the Reynolds number in the current study is limited to $2.0 \times 10^{6}$ at $4.5 \mathrm{~m} / \mathrm{s}$ due to concerns about exceeding the load limit of the model. The subscript $\infty$ denotes freestream conditions.

\subsection{High-Speed Proportional Valves}

Due to the lack of commercially-available high-frequency proportional valves for water, two custom-made valves are purposely constructed (see Fig. 2). The valves build on the body of Danfoss EV210B, where the electromagnetic coils are lengthened and the core tubes are extended and modified. To keep the valves from overheating, each electromagnetic coil is wrapped in a water cooling coil, which in turn is encased in thermally conductive epoxy. The valves allow high flow rates of up to $3 \mathrm{l} / \mathrm{s}$ and actuation frequencies of up to $30 \mathrm{~Hz}$. The flow rate fluctuation levels are however dependent on the actuation frequency. The blowing intensity amplitude decreases with frequency as the core tube inertia becomes increasingly difficult to overcome. This amplitude drop can be compensated (to some extent) by increasing the power. The valves are controlled by a special electronic circuit, illustrated in Fig. 2. A signal produced by a signal generator is sent to a pulse width modulation (PWM) unit and then to a solid state relay. This electronic circuit is a cheaper alternative to using massive amplifiers, albeit at the cost of restricting the signal to a rectangular train type. An adjustable $(0$ to $60 \mathrm{~V})$ voltage power source provides the necessary power. To minimize hydraulic dampening, the two valves are attached on either side of the airfoil model close to both plenum inlets (see Fig. 1). Two Prandtl probes installed between the valves and the model plenum are used to measure the flowrate and thus the actuation intensity in real time.

\subsection{Instrumentation and Measurement Technique}

Since the main objective of this work is to examine the possible lift gains brought by superimposing periodic actuation, all presented results are surface pressure measurements and corresponding lift coefficients. The pressure distributions are measured using 64 pressure taps along the mid-span section connected to high precision $(0.1 \%$ FS error) Keller PD-X33 pressure transducers. Each ensemble is sampled at $100 \mathrm{~Hz}$ 


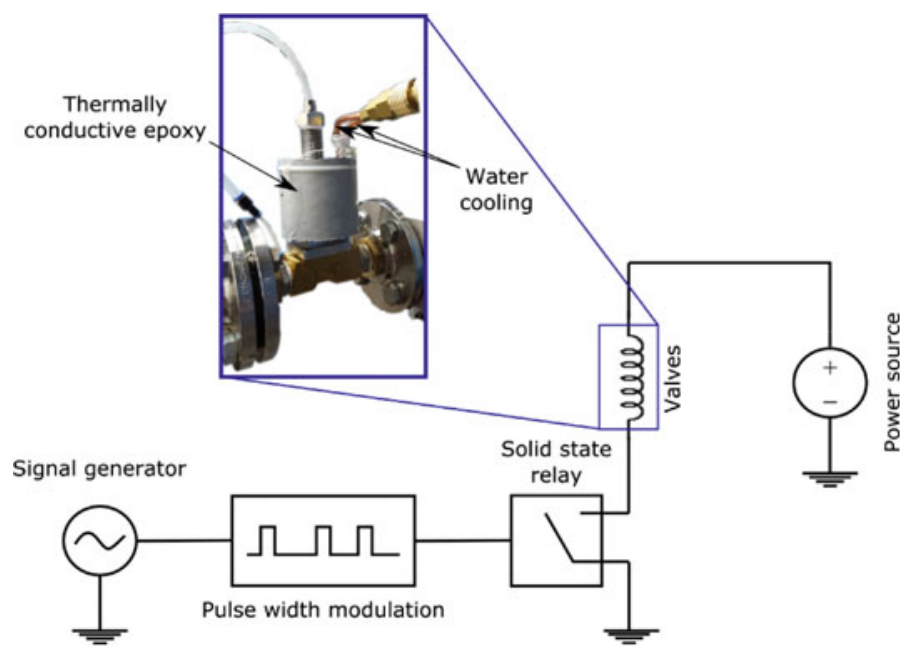

Fig. 2 Schematic of the electronic circuit controlling the two in-house constructed proportional valves, along with a picture of one of the valves

for $5 \mathrm{~s}$. Besides the pressure taps, the model is instrumented with seven real-time miniature piezo-resistive pressure Keller sensors with $300 \mathrm{kHz}$ possible eigenfrequency that are flush mounted onto the Coanda flap. These real-time pressure sensors will provide the input for the upcoming closed-loop control measurement campaign.

\subsection{Test Cases}

Periodic actuation is characterized by three parameters, the actuation frequency, the blowing intensity, and the blowing amplitude. The actuation frequency $f^{a}$ is usually presented in its non-dimensional form $F^{+}$, defined as [1]

$$
F^{+}=\frac{f^{a} c_{f l}}{V_{\infty}},
$$

where $c_{f l}$ is the flap chord length. In this study, the actuation frequency can reach a maximum of 1 at $\operatorname{Re}=1 \times 10^{6}$, imposed by the valves mechanical limitations. The blowing intensity of a circulation control wing is usually characterized by the non-dimensional momentum coefficient $c_{\mu}$ [17]. The momentum coefficient was first introduced by Poisson-Quinton and Lepage [22] as the blown jet thrust normalized by the product of the free stream dynamic pressure and a reference area

$$
c_{\mu}=\frac{\rho_{j e t} V_{j e t}^{2} h}{\frac{1}{2} \rho_{\infty} V_{\infty}^{2} S_{r e f}},
$$


where $V_{j e t}$ is the jet nozzle average exit velocity, $h$ is the slit height, and $S_{\text {ref }}$ is the projected clean wing area. The momentum coefficient, in this case, contains both the mean and the periodic component. For sinusoidal actuation, the momentum coefficient can be expressed as

$$
c_{\mu}=C_{\mu}+C_{\mu 1} \cos \left(2 \pi f^{a} t\right)=C_{\mu}+C_{\mu 1} \cos \left(\frac{2 \pi V_{\infty}}{c_{f l}} F^{+} t\right),
$$

where $C_{\mu}$ is the mean steady component, and $C_{\mu 1}$ is the oscillation amplitude.

The measurements are acquired for a range of momentum coefficients, starting from the natural unactuated case $C_{\mu}=0$ and up to $C_{\mu}=0.06$, where the flow is fully attached to the flap. Measurements are conducted for three Reynolds numbers $\operatorname{Re}=1 \times 10^{6}, 1.5 \times 10^{6}$, and $2.0 \times 10^{6}$ and for angles of attack up to $22^{\circ}$.

\section{Methodology}

\subsection{Identification of Sinusoidal Actuation}

The type of actuation signal (e.g. sinusoidal, square, etc) can affect the flow's aerodynamic response. With a square wave voltage input from the PWM, the custom-built valves are designed to deliver sinusoidal forcing. This sinusoidal flow results from viscous dampening effects of the flow, and from the valve mechanism itself: the coil cannot react instantly to the square voltage input, as it requires a finite time to build a strong-enough magnetic field to push the rod. The valves operate in fully-open mode by default. When the magnetic coils are energized, the core rods are pushed downward to constrict the flow. The closing force is dependent on the actuation frequency (rod inertia) and on the pressure acting against the rod (blowing intensity). For certain actuation frequency/pressure settings, the rod/flow interactions yield non-sinusoidal flow rates (and hence momentum coefficients). Figures $3 \mathrm{a}$ and $4 \mathrm{a}$ show one sinusoidal and one non-sinusoidal time trace of the momentum coefficient, respectively. Non-sinusoidal forcing occurs in $\approx 13 \%$ of the total number of test cases, and yield a different aerodynamic response than that of the sinusoidal one. This motivated the identification and deletion of non-sinusoidal actuation signals. The procedure involves four steps:

1. Fourier transform the momentum coefficient trace, $\mathcal{F}\left(c_{\mu}\right)$ (e.g. Figs. $3 b$ and $4 b$ ).

2. Identify the peaks in the Fourier transform.

3. Compute the ratio $\mathfrak{R}$ between the highest peak and the second highest nonharmonic peak.

4. Enforce a $\mathfrak{R}>5$ threshold for sinusoidal classification. 

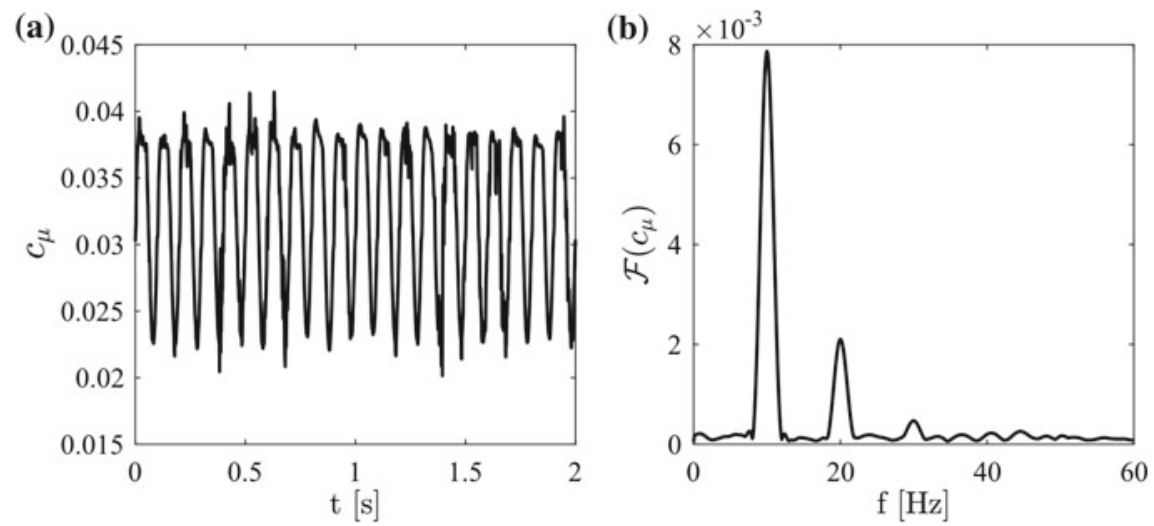

Fig. 3 a A sinusoidal time trace of the momentum coefficient, and $\mathbf{b}$ its corresponding Fourier transform
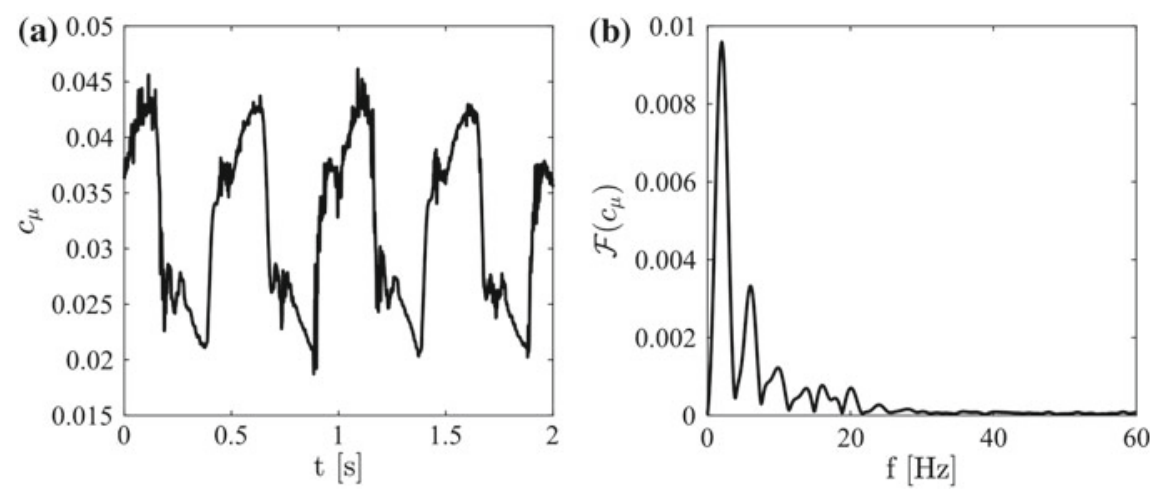

Fig. 4 a A non-sinusoidal time trace of the momentum coefficient, and $\mathbf{b}$ its corresponding Fourier transform

All momentum coefficient signals with $\mathfrak{R}<5$ are thus considered non-sinusoidal and are removed. The threshold is simply reached by trial and error. The Fourier transformation peak also served to identify the actuation amplitude $C_{\mu 1}$ in Eq. (3).

\subsection{Sparse Modeling of the Lift Gains/Losses}

This section describes the methodology to identify a sparse nonlinear model of the lift gains/losses. The motivation behind promoting model sparsity is to reduce complexity and to avoid overfitting. The current approach is comparable to the sparse identification of nonlinear dynamical systems (SINDY) method [23], but for a response surface. Specifically, we are concerned with identifying a sparse nonlinear model 
for the lift gains/losses based on the experimentally-measured data for a range of actuation parameters $C_{\mu}, C_{\mu 1}$ and $F^{+}$(refer to Eq.3). In other words, we seek to identify the following model

$$
\Delta \mathbf{C}_{\mathbf{l}}=\Delta \mathbf{C}_{\mathbf{l}}(\mathbf{X})
$$

where,

$$
\Delta \mathbf{C}_{\mathbf{l}}=\left[\begin{array}{c}
\Delta C_{l, 1} \\
\Delta C_{l, 2} \\
\vdots \\
\Delta C_{l, m}
\end{array}\right]
$$

are the lift gains/losses for $m$ test cases, and

$$
\mathbf{X}=\left[\begin{array}{c}
x_{1} \\
x_{2} \\
\vdots \\
x_{m}
\end{array}\right]=\left[\begin{array}{ccc}
\hat{C}_{\mu, 1} & \hat{C}_{\mu 1,1} & \hat{F}_{1}^{+} \\
\hat{C}_{\mu, 2} & \hat{C}_{\mu 1,2} & \hat{F}_{2}^{+} \\
\vdots & \vdots & \vdots \\
\hat{C}_{\mu, m} & \hat{C}_{\mu 1, m} & \hat{F}_{m}^{+}
\end{array}\right]
$$

are the normalized actuation settings, $\hat{C}_{\mu}=\frac{C_{\mu}}{\max \left\{C_{\mu}\right\}}, \hat{C}_{\mu 1}=\frac{C_{\mu 1}}{\max \left\{C_{\mu 1}\right\}}, \hat{F}^{+}=\frac{F^{+}}{\max \left\{F^{+}\right\}}$. The normalization ensures that

$$
-1 \leq \hat{C}_{\mu}, \hat{C}_{\mu 1}, \hat{F}^{+} \leq 1
$$

a condition that greatly simplifies the sparse optimization problem in the identification process. Modeling begins by constructing an augmented library $\Theta(\mathbf{X})$ consisting of candidate nonlinear functions of the columns of $\mathbf{X}$. For example, $\Theta(\mathbf{X})$ may consist of constant and polynomial terms:

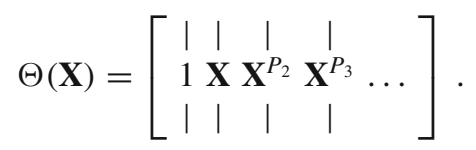

Here, $\mathbf{X}^{P_{i}}$ denote higher polynomials. For example, $\mathbf{X}^{P_{2}}$ denotes the quadratic nonlinearities in $\mathbf{X}$, given by:

$$
\mathbf{X}^{P_{2}}=\left[\begin{array}{ccccccc}
\hat{C}_{\mu, 1}^{2} & \hat{C}_{\mu, 1} \hat{C}_{\mu 1,1} & \hat{C}_{\mu, 1} \hat{F}_{1}^{+} & \hat{C}_{\mu 1,1}^{2} & \hat{C}_{\mu 1,1} \hat{F}_{1}^{+} & \hat{F}_{1}^{+^{2}} \\
\hat{C}_{\mu, 2}^{2} & \hat{C}_{\mu, 2} \hat{C}_{\mu 1,2} & \hat{C}_{\mu, 2} \hat{F}_{2}^{+} & \hat{C}_{\mu 1,2}^{2} & \hat{C}_{\mu 1,2} \hat{F}_{2}^{+} & \hat{F}_{2}^{+^{2}} \\
\vdots & \vdots & \vdots & \vdots & \vdots & \vdots \\
\hat{C}_{\mu, m}^{2} & \hat{C}_{\mu, m} \hat{C}_{\mu 1, m} & \hat{C}_{\mu, m} \hat{F}_{m}^{+} & \hat{C}_{\mu 1, m}^{2} & \hat{C}_{\mu 1, m} \hat{F}_{m}^{+} \hat{F}_{m}^{+^{2}}
\end{array}\right]
$$


For the current study, the highest polynomial order is set to four. Hence,

$$
\Theta(\mathbf{X})=\left[\begin{array}{ccccc}
\mid & \mid & \mid & \mid & \mid \\
1 & \mathbf{X} & \mathbf{X}^{P_{2}} & \mathbf{X}^{P_{3}} & \mathbf{X}^{P_{4}} \\
\mid & \mid & \mid & \mid & \mid
\end{array}\right]
$$

Each column of $\Theta(\mathbf{X})$ represents a candidate function for the model in Eq. (4). There is a large number of possible entries in this matrix of nonlinearities. Since we are seeking a parsimonious model, only a few of these nonlinearities are active. Moreover, the measured lift coefficient contains experimental uncertainties. The solution to this overdetermined system with noise is obtained by solving

$$
\Delta \mathbf{C}_{\mathbf{l}}=\Theta(\mathbf{X}) \Xi+\eta \mathbf{Z},
$$

which yields the sparse vectors of coefficients $\Xi=\left[\xi_{1} \xi_{2} \ldots \xi_{n}\right]$. Here, $\mathbf{Z}$ represents white noise with vanishing mean and unit variance while $\eta$ denotes noise gain. System (10) can be easily solved using the LASSO [24], which is an $\ell_{1}$-regression that promotes sparsity. The LASSO solves the minimization problem,

$$
\hat{\beta}^{\text {lasso }}=\operatorname{argmin}_{\beta}\left\{\frac{1}{2} \sum_{i=1}^{m}\left(y_{i}-\beta_{0}-\sum_{j=1}^{p} x_{i j} \beta_{j}\right)^{2}+\lambda \sum_{j=1}^{p} \beta_{j}\right\}
$$

where $y_{i}$ is the response $\left(\Delta C_{l i}\right)$ at observation $i, \lambda$ is a positive regularization parameter, and $\beta_{0}$ and $\beta$ are the model coefficients. Hence, as $\lambda$ increases, the number of nonzero components of $\beta$ decreases. To select the proper regularization parameter, we examine the cross-validated mean-square error of the model for a range of $\lambda$ 's (Fig. 5). As expected, the mean square error reduces with smaller values of $\lambda$, which

Fig. 5 The cross-validated mean square error for a range of $\lambda$ 's

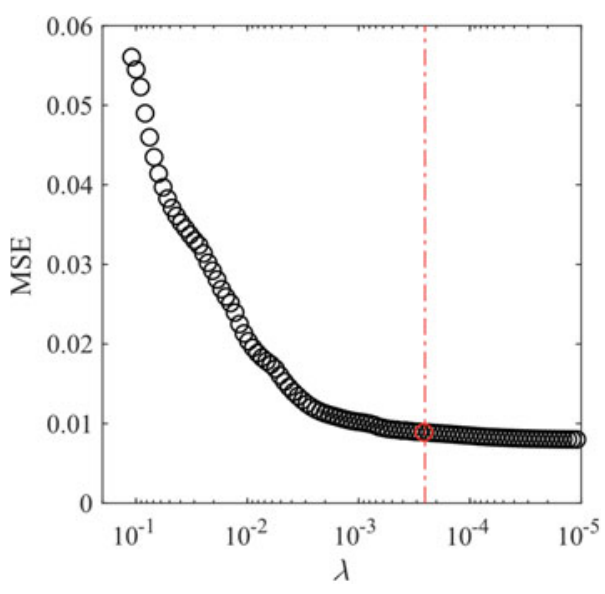


also indicate higher model complexity. The red circle and dashed line indicate the selected regularization parameter $\lambda$, which yields fifteen nonactive coefficients from a total of thirty five, and a mean-square error of $\approx 0.008$. This selection is a compromise between complexity and accuracy. For $\alpha=0^{\circ}$ and $\operatorname{Re}=1.5 \times 10^{6}, 706$ measured test cases covering four mean steady momentum coefficients $\left(C_{\mu}=0.03-0.05\right)$ are used to train the model, which reads

$$
\begin{aligned}
\Delta C_{l}= & 0.1874+2.8211 \hat{C}_{\mu, 1}-0.0443 \hat{F}^{+}-0.3891 \hat{C}_{\mu}-2.2557 \hat{C}_{\mu, 1} \hat{F}^{+} \\
& -0.0232 \hat{C}_{\mu, 1} \hat{C}_{\mu}-0.5286 \hat{C}_{\mu, 1}^{3}-1.1201 \hat{C}_{\mu, 1}^{2} \hat{C}_{\mu}-6.2910 \hat{C}_{\mu, 1} \hat{F}^{+2} \\
& -2.0115 \hat{C}_{\mu, 1} \hat{C}_{\mu}^{2}+0.0972 \hat{F}^{+}+0.0043 \hat{F}^{+} \hat{C}_{\mu}^{2}+0.7102 \hat{C}_{\mu, 1}^{3} \hat{F}^{+} \\
& +1.9343 \hat{C}_{\mu, 1}^{2} \hat{F}^{+} \hat{C}_{\mu}+4.7541 \hat{C}_{\mu, 1} \hat{F}^{+3}-0.6339 \hat{C}_{\mu, 1} \hat{F}^{+2} \hat{C}_{\mu} \\
& +4.8698 \hat{C}_{\mu, 1} \hat{F}^{+} \hat{C}_{\mu}^{2}-0.6847 \hat{C}_{\mu, 1} \hat{C}_{\mu}^{3}-0.0002 \hat{F}^{+2} \hat{C}_{\mu}^{2}+0.1589 \hat{C}_{\mu}^{4}
\end{aligned}
$$

\section{Results}

\subsection{Steady Actuation}

The pressure distributions over the airfoil with increasing blowing intensity are shown in Fig. 6 for zero angle of attack and $\mathrm{Re}=1.0 \times 10^{6}$ and $\mathrm{Re}=1.5 \times 10^{6}$, respectively. The benefit of the droop nose is clear. The low and rounded suction peak at the leading edge reduces the adverse pressure gradient and thereby enhances the boundary layer over the suction side and its receptivity to actuation. This in turn

(a)

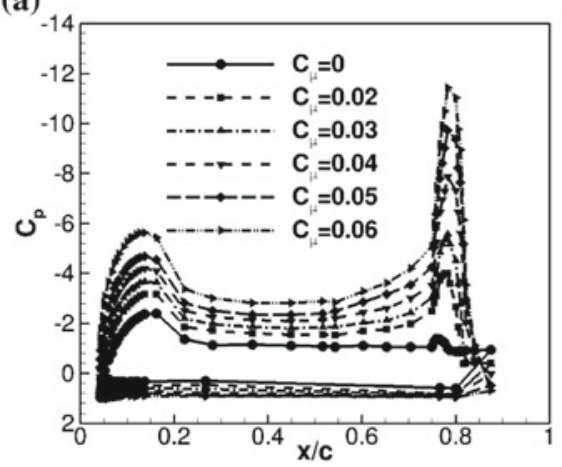

(b)

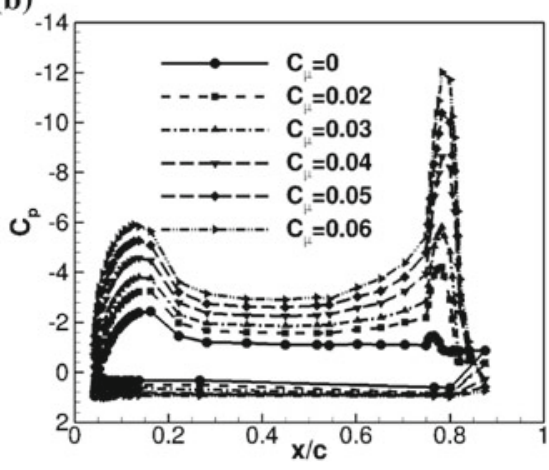

Fig. 6 Pressure coefficient distribution with increasing steady momentum coefficients for $\mathbf{a} \operatorname{Re}=1.0 \times 10^{6}$ and $\mathbf{b} \operatorname{Re}=1.5 \times 10^{6}$ at $\alpha=0^{\circ}$ 

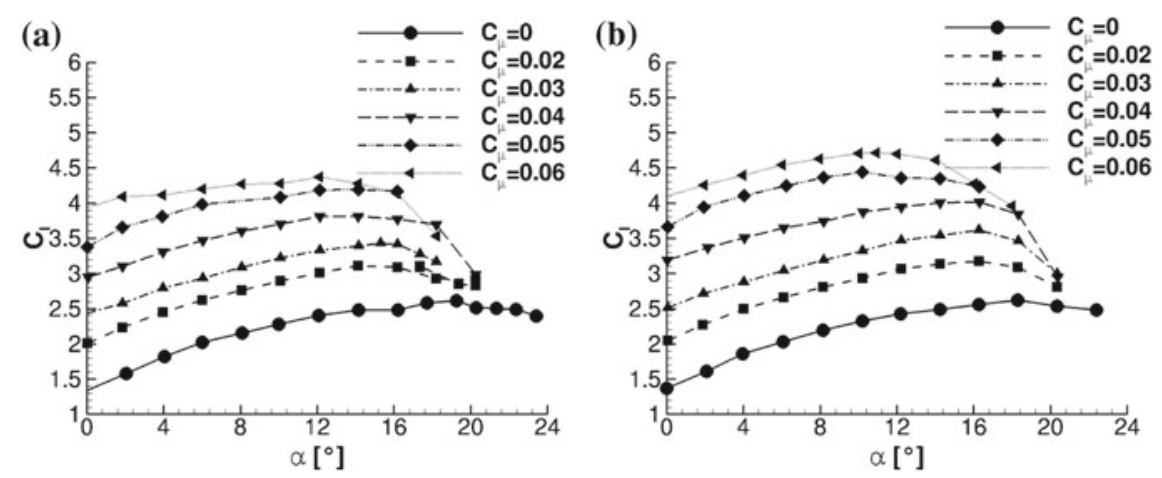

Fig. 7 The effect of the steady momentum coefficient on the lift coefficient over a range of angles of attack at $\mathbf{a} \operatorname{Re}=1.0 \times 10^{6}$ and $\mathbf{b} \operatorname{Re}=1.5 \times 10^{6}$, respectively

yields improved aerodynamic performance, such as increased lift and higher stall angles compared to a traditional droop nose [25].

The second suction peak near the trailing edge results from the locally high rate of flow turning, which is enabled the jet momentum injection that increases with higher blowing intensities. Stronger actuation also increases the circulation, shifting the stagnation point away from the leading edge over the pressure side. The increase in actuation causes the separation to gradually recede over the flap up to $C_{\mu}=0.05$ where the flow becomes fully attached. This gradual mitigation of separation and the added circulation result in steady lift gains, as shown in Fig. 7 for six blowing intensities at two Reynolds numbers. The lower stall angles with higher $C_{\mu}$ can be also observed. Figure 7 also shows the Reynolds number influence on the aerodynamic performance, with higher Reynolds numbers yielding higher lift coefficients. Further increase in the Reynolds number yields smaller lift gains (not shown).

\subsection{Unsteady Actuation}

The lift gains/losses distributions from superimposing a periodic component onto the steady actuation are presented in Fig. 8 for two test cases at $\mathrm{Re}=1.5 \times 10^{6}$ and $\alpha=0^{\circ}$ with mean blowing intensities of $C_{\mu}=0.03$ and $C_{\mu}=0.05$, respectively. The test cases are selected for their different aerodynamic characteristics (see Figs. 6 and 7), where the flow is partially separated for $C_{\mu}=0.03$ and is attached for $C_{\mu}=0.05$ during steady actuation $\left(F^{+}=0\right)$. The contour distributions are those of the modeled $\Delta C_{l}$, and the dots designate the measured test cases. The addition of a periodic component to actuation has an opposite effect on the two cases. For the $C_{\mu}=0.03$ case, unsteady forcing yields lift increases throughout the tested actuation frequency and amplitude range. The highest lift gains with respect to the reference steady case is $\Delta C_{l}=0.47$ achieved at $F^{+}=0.17$ and $C_{\mu 1}=0.016$. On the other hand, unsteady actuation is detrimental to the lift coefficient for the $C_{\mu}=0.05$ case almost across 

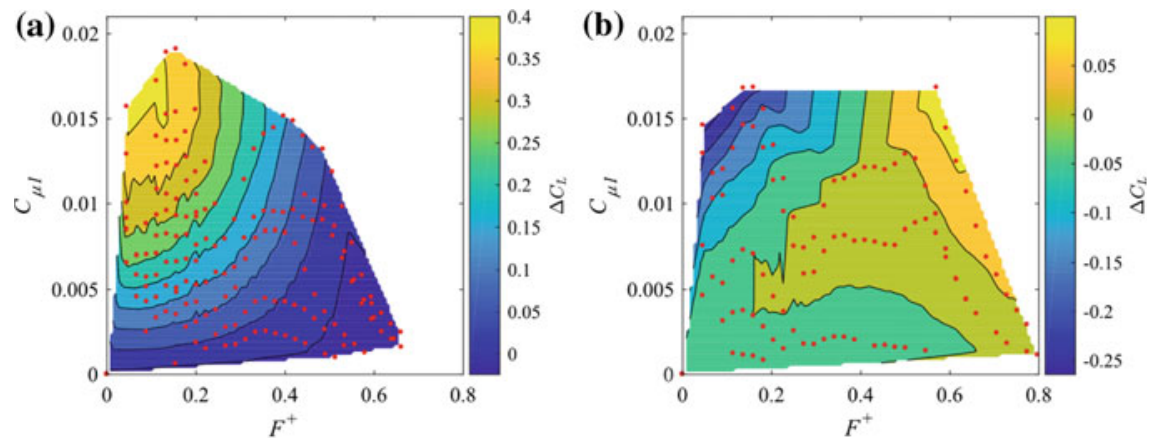

Fig. 8 Modeled $\Delta C_{l}$ distributions for a range of actuation amplitudes $C_{\mu 1}$ and actuation frequencies $F^{+}$for $\mathbf{a} C_{\mu}=0.03$ and $\mathbf{b} C_{\mu}=0.05$ at $\mathrm{Re}=1.5 \times 10^{6}$

the entire actuation parameter range. In fact, the lift coefficient decreases by as much as $\Delta C_{l}=-0.3$ at $F^{+}=0.11$ and $C_{\mu 1}=0.015$.

The evolution of the lift gain with increasing momentum coefficient can be examined using the previously-developed model. Figure 10 shows the lift gain distribution over a range of $C_{\mu}$ for a fixed actuation amplitude $C_{\mu 1}=0.016$ and a fixed actuation frequency $F^{+}=0.11$. As the figure shows, the lift gains gradually decrease with increasing momentum coefficient until they become lift losses starting $C_{\mu} \approx 0.04$, when the flow starts to fully attach. The highest lift gains are achieved at the lowest evaluated mean steady momentum coefficient, where the flow is partially attached. Since there exist no measurements at lower $C_{\mu}$, it is not clear if these lift gains continue their increase. It is worth to note that even if the lift gains were highest at lower $C_{\mu}$, the overall lift is low and is of no interest to a STOL-capable aircraft.

Figure 9 presents the modeled $\Delta C_{l}$ distributions for a range of actuation amplitudes $C_{\mu 1}$ and actuation frequencies $F^{+}$for (a) $\alpha=0^{\circ}$ and (b) $\alpha=12^{\circ}$ at $C_{\mu}=0.035$ and $\operatorname{Re}=1.5 \times 10^{6}$. Compared to Fig. $8 \mathrm{a}$, the two distributions exhibit a similar shape
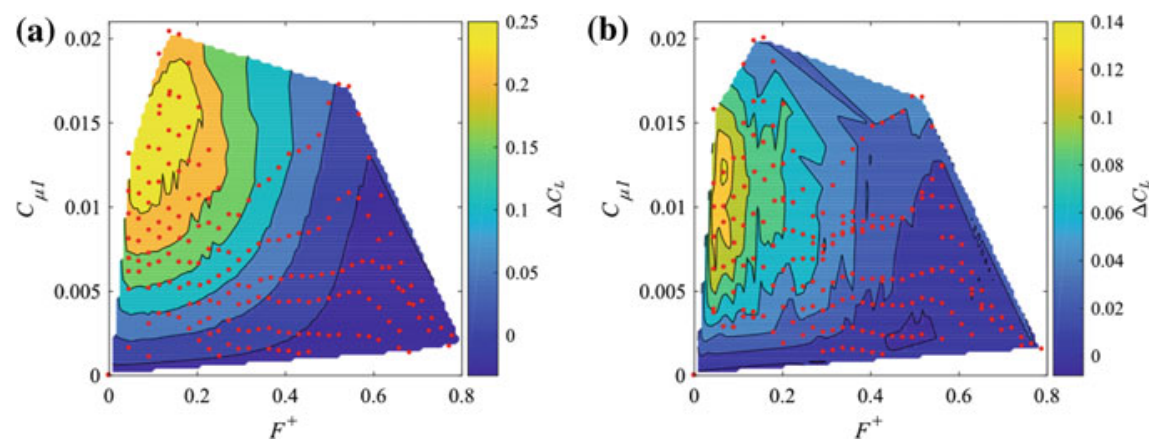

Fig. 9 Modeled $\Delta C_{l}$ distributions for a range of actuation amplitudes $C_{\mu 1}$ and actuation frequencies $F^{+}$for $\mathbf{a} \alpha=0^{\circ}$ and $\mathbf{b} \alpha=12^{\circ}$ at $C_{\mu}=0.035$ and $\operatorname{Re}=1.5 \times 10^{6}$ 
Fig. 10 The evolution of $\Delta C_{l}$ over a range of blowing intensities for $C_{\mu 1}=0.016$ and $F^{+}=0.11$ at $\alpha=0^{\circ}$

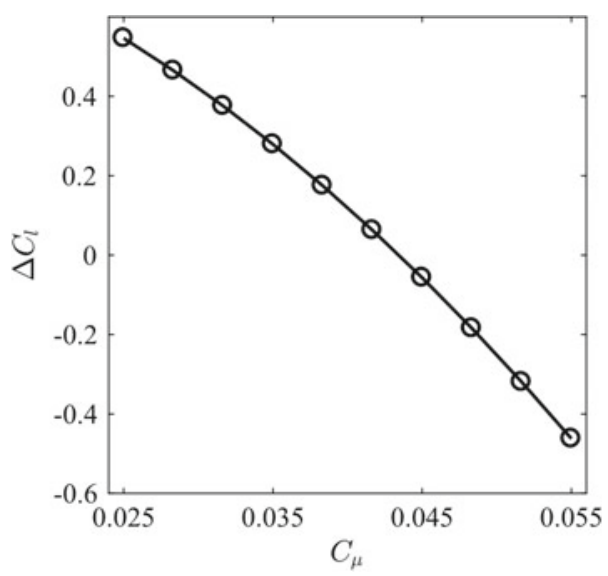

with the highest lift gains at $F^{+} \approx 0.1$. However, between $\alpha=0^{\circ}$ and $\alpha=12^{\circ}$ the maximum lift gains are halved from $\Delta C_{l}=0.3$ to 0.14 . This reduction in lift gains is attributed to uncertainty in measuring the lift coefficient for the steady-blown cases, as shown in Fig. 11, where a comparison between an actuated $\left(F^{+}=0.11\right.$ and $\left.C_{\mu 1}=0.012\right)$ and the non-dynamically actuated $\left(F^{+}=0\right)$ lift coefficient over a range of angles of attack is shown. As can be seen, the lift gains $\left(\Delta C_{l}\right)$ remain constant up to $\alpha=10^{\circ}$, followed by a small drop starting at $12^{\circ}$. This small drop in lift gain is caused by an unexpected small increase in the lift coefficient for the steady blown case. We suspect that this small increase is an anomaly in the measurements, which will be addressed in our next campaign.

For all cases, the maximum lift gains occur at amplitudes smaller than the largest measured one and smaller than the corresponding mean momentum coefficient $C_{\mu}$.

Fig. $11 C_{l}$ versus $\alpha$ for an actuated and a non-dynamically actuated case for $C_{\mu}=0.035$ and $\operatorname{Re}=1.5 \times 10^{6}$

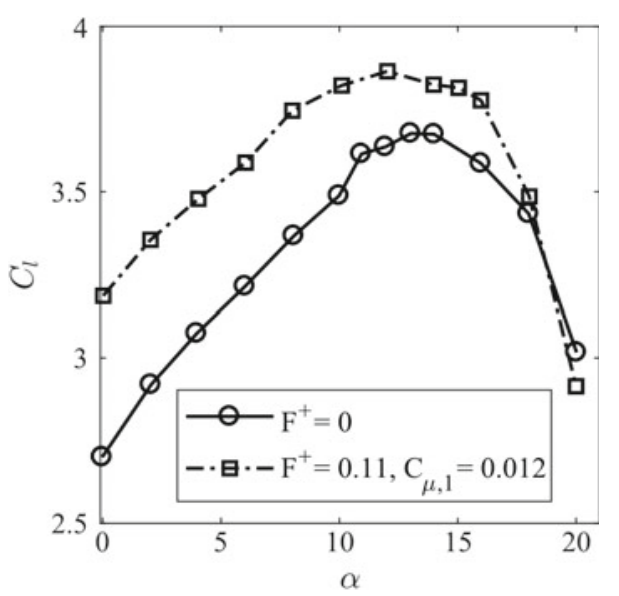


Hence, the maximum aerodynamic gains are achieved at amplitudes that do not require complete closing/opening of the valves. This demonstrates the advantage of having actuation with independently-controlled, steady and unsteady components, something switching valves cannot perform. Moreover, the highest lift gains are achieved at an actuation frequency of $F^{+} \approx 0.1$, which is not harmonically related to the natural shedding frequency of the flow, $S t=\frac{f \cdot \mathcal{c}_{f}}{V_{\infty}}=0.23$ [16]. It is not entirely clear what the significance of this frequency is. Without any field wake measurements and spectral analysis, it is difficult to accurately pinpoint the physical mechanisms yielding the relative lift increase. However, based on previous studies [1, 19] and current observations, it is possible to infer the underlying phenomena. Separated or partially-separated flows are more receptive to unsteady actuation targeting some flow instabilities. Here, the actuation frequency can excite or dampen certain mode(s) to delay separation and to increase circulation. On the other hand, periodic actuation on attached flows can only disturb the attachement without significant benefits. Moreover, oscillations associated with dynamic actuation generate additional vorticity in the flow field contributing to adverse changes in lift and drag. Attached flows can only attain higher lift through added circulation, which cannot be provided by periodic forcing. Time-resolved PIV measurements are planned in a future campaign to confirm these hypotheses.

\section{Conclusion}

This study investigates and models the lift gains and losses from superimposing a periodic actuation component on a steady component for a high-lift configuration with a highly deflected Coanda flap. The assessment relies on pressure measurements along the model centerline. Steady actuation is shown to increase the lift coefficient significantly up to $C_{l, \max }=4.72$ at $\operatorname{Re}=1.5 \times 10^{6}$. This high lift coefficient enables short take-off and landing (STOL) capabilities. Further lift gains are achieved through the superposition of a periodic forcing component. For test cases where the flow is partially separated during steady actuation, superimposed periodic forcing yields lift increases through most of the actuation parameter range. The highest lift gain with respect to the reference steady case reaches $\Delta C_{l}=0.47$. On the other hand, superimposed periodic actuation is detrimental to the lift coefficient for attached flows through most of the actuation parameter range. In fact, the lift coefficient decreases by as much as $\Delta C_{l}=-0.3$. The highest aerodynamic gains are achieved at amplitudes that do not require complete closing/opening of the valves, demonstrating the advantage of having actuation with independently-controlled steady and unsteady components. The highest gains are also achieved at an actuation frequency of $F^{+} \approx 0.1$, which is not harmonically related to the natural shedding frequency of the flow. Further investigations and time-resolved PIV measurements are required to understand the underlying phenomena leading to the lift increase/decrease. 
Acknowledgements We acknowledge the funding of the Collaborative Research Centre (CRC 880) "Fundamentals of High Lift of Future Civil Aircraft" supported by the Deutsche Forschungsgemeinschaft (DFG) and hosted at the Technische Universität Carolo-Wilhelmina zu Braunschweig.

\section{References}

1. Greenblatt, D., Wygnanski, I.: The control of flow separation by periodic excitation. Prog. Aerosp. Sci. 36(7), 487-545 (2000)

2. Barros, D., Borée, J., Noack, B.R., Spohn, A., Ruiz, T.: Bluff body drag manipulation using pulsed jets and Coanda effect. J. Fluid Mech. 805, 422-459 (2016)

3. Chabert, T., Dandois, J., Garnier, A.: Experimental closed-loop control of separated-flow over a plain flap using extremum seeking. Exp. Fluid 57(37) (2016)

4. Davidson, I.M.: Aerofoil boundary layer control system. Brit. Pat. 913, 754 (1960)

5. Lachmann, G.V.: Boundary Layer and Flow Control: Its Principles and Application. Pergamon Press, New York (1961)

6. Wood, N.J., Nielsen, J.N.: Circulation control airfoils-Past, present and future. In: AIAA Paper-85, p. 0204 (1985)

7. Englar, R.: Circulation control pneumatic aerodynamics: blown force and moment augmentation and modifications; past, present, and future. In: AIAA Paper-2000, p. 2541 (2000)

8. Sexstone, M.G., Huebner, L.D., Lamar, J.E., McKinley, R.E., Torres, A.O., Burley, C.L., Scott, R.C., Small, W.J.: Synergistic airframe-propulsion interactions and integrations. Technical Report No. NASA/TM-1998-207644. NASA Langley Research Center; Hampton, VA United States (1998)

9. Smith, A.M.O.: High-lift aerodynamics. J. Aircr. 12(6), 501-530 (1975)

10. Jensch, C., Pfingsten, K.C., Radespiel, R., Schuermann, M., Haupt, M., Bauss, S.: Design aspects of a gapless high-lift system with active blowing. In: Deutscher Luft- und Raumfahrtkongress, vol. 58, Aachen, Germany (2009)

11. Radespiel, R., Pfingsten, K.-C., Jensch, C.: Flow analysis of augmented high-lift systems. In: Radespiel, R., Rossow, C.C., Brinkmann, B.W. (eds.) Hermann Schlichting-100 Years, pp. 168-189 (2009)

12. Radespiel, R., Burnazzi, M., Casper, M., Scholz, P.: Active flow control for high lift with steady blowing. Aeronaut. J. 120(1223), 171-200 (2016)

13. Oyler, T.E., Palmer, W.E.: Exploratory Investigation of Pulse Blowing for Boundary Layer Control. Technical Report No.: NR72H-12, North American Rockwell, Columbus division (1972)

14. Walters, R.E., Myer, D.P., Holt, D.J.: Circulation Control by Steady and Pulsed Blowing for a Cambered Elliptical Airfoil. Technical Report No.:TR-32, West Virginia University, Aerospace Engineering (1972)

15. Jones, G.S., Viken, S.A., Washburn, A.E., Jenkins, L.N., Cagle, C.M.: An active flow circulation controlled flap concept for general aviation aircraft applications. In: AIAA paper-2002, p. 3157 (2002)

16. El Sayed, Y., Semaan, R., Sattler, S., Radespiel, R.: Wake characterization methods of a circulation control wing. Exp. Fluids 58(10), 144 (2017)

17. El Sayed, M.Y., Beck, N., Kumar, P., Semaan, R., Radespiel, R.: Challenges in the experimental quantification of the momentum coefficient of circulation controlled wings. In: New Results in Numerical and Experimental Fluid Mechanics XI, pp. 533-543. Springer (2018)

18. Petz, R., Nitsche, W.: Active separation control on the flap of a two-dimensional generic highlift configuration. J. Aircr. 44(3), 865-874 (2007)

19. Seifert, A., Greenblatt, D., Wygnanski, I.J.: Active separation control: an overview of Reynolds and Mach number effects. Aerosp. Sci. Technol. 8(7), 569-582 (2004) 
20. Burnazzi, M., Radespiel, R.: Design and analysis of a droop nose for coanda flap applications. AIAA J. Aircr. 51(5), 1567-1579 (2014)

21. Scholz, P., Sattler, S., Wulff, D.: Der Große Wasserkanal 'GWB'-Eine Versuchsanlage für zeitauflösende Messungen bei großen Reynoldszahlen. Deutscher Luft-und Raumfahrtkongress, Stuttgart, Germany (2013)

22. Poisson-Quinton P., Lepage L.: Survey of French research on the control of boundary layer and circulation. In: Lachmann, G.V. (eds.) Boundary layer and Flow Control: Its Principles and Application, vol. 1, pp. 21-73 (1961)

23. Brunton, S.L., Proctor, J.L., Nathan, J.K.: Discovering governing equations from data by sparse identification of nonlinear dynamical systems. Proc. Natl. Acad. Sci. 113(15), 3932-3937 (2016)

24. Tibshirani, R.: Regression shrinkage and selection via the lasso. J. Roy. Stat. Soc. Ser. B (Methodol), 267-288 (1996)

25. Burnazzi, M., Radespiel, R.: Assessment of leading-edge devices for stall delay on an airfoil with active circulation control. CEAS Aeronaut. J. 5(4), 359-385 (2014) 